\title{
Feedforward Inhibition in Biosynthetic Pathways: Inhibition of the Aminoacyl-tRNA Synthetase by the Penultimate Product
}

\author{
Michael A. Savageau \\ Department of Microbiology, University of Michigan, \\ Ann Arbor, Michigan 48109, U.S.A.
}

(Received 18 January 1978, and in revised form 9 May 1978)

Inhibition of the aminoacyl-tRNA synthetase by the penultimate product of a pathway for the biosynthesis of an amino acid has been reported for several pathways in many different types of organisms. A regulatory role for this mechanism often has been suggested, although there is some conflicting experimental evidence. The significance of such feedforward inhibition is examined here by mathematical analysis. The techniques that have proved successful in the analysis of control by feedback inhibitionshowing that the nearly universal pattern of end-product inhibition is an optimal design-indicate that feedforward inhibition by the penultimate product does not contribute significantly to the functional effectiveness of regulation at the level of enzymatic activity. Feedforward inhibition by the penultimate product may have no physiological role, or it may be involved in differential signalling of intra- and extracellular changes and/or in directing the metabolic flow in branched pathways. These possibilities are discussed in light of analytical results presented in this paper and published experimental evidence.

\section{Introduction}

Feedforward inhibition, in which the penultimate product of a biosynthetic pathway for an amino acid inhibits the aminoacyl-tRNA synthetase further ahead in the pathway, may represent a novel control mechanism at the level of enzymatic activity. This phenomenon was first reported by Ames \& Hartman (1961) in the histidine biosynthetic pathway of Salmonella typhimurium. It was noted and studied more extensively in the arginine biosynthetic pathway of Neurospora crassa by Nazario (1967). Sussenbach \& Strijkert (1969) also found this mechanism in the arginine biosynthetic pathway of Chlamydomonas reinhardi and were the first to attach regulatory significance to it. These observations have been repeated in Escherichia coli by Williams et al. (1973).

385 
More recently, Charlier \& Gerlo (1976) examined the regulation of the arginine biosynthetic pathway in E. coli and were unable to detect inhibition of partially purified arginyl-tRNA synthetase by the penultimate product argininosuccinate. In studies of the same system from Neurospora, Barthelmess, Curtis \& Kacser (1974) found no evidence in viro of such feedforward inhibition, while Spurgeon \& Matchett (1977) reported evidence of it both in vivo and in vitro.

In view of this conflicting evidence, it would be desirable to have a detailed analysis that might uncover functional implications of this mechanism. For example, by means of such analysis, it was shown that end-product inhibition is an optimal design for feedback control of biosynthetic pathways (Savageau, 1974, 1975). A preliminary analysis suggested some functional roles for feedforward inhibition operating together with feedback inhibition and in branched pathways (Savageau, 1972). In the following pages a more complete analysis, based on several different criteria for functional effectiveness, will be given. The results show few advantages of feedforward inhibition by the penultimate product. These conclusions are discussed in the light of analytical results presented in this paper and available experimental evidence.

\section{Method of Analysis}

The general model of a biosynthetic pathway represented in Fig. 1, including both feedback and feedforward interactions, will be analyzed. The descriptive equations for this model can be written in a non-linear

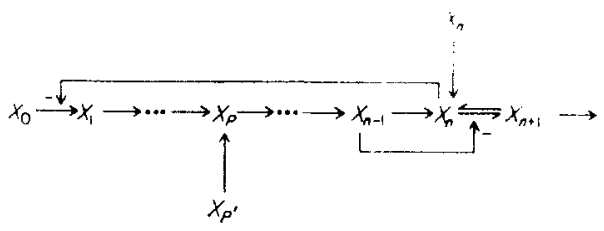

Fig. 1. Model of an unbranched pathway for the biosynthesis of an amino acid, including feedback inhibition of the first enzyme by the amino acid end product and feedforward inhibition of the aminoacyl-tRNA synthetase by the penultimate product. $X_{i}$ represents the $i$ th metabolite, $X_{p}{ }^{\prime}$, and $X_{n}{ }^{\prime}$, represent extracellular pools corresponding to the intracellular pools of an intermediate $X_{p}$ and the end product $X_{n}$, respectively, and $X_{n+1}$ represents aminoacyl tRNA, the activated end product. An arrow between symbols represents an enzyme-catalyzed reaction or transport process that is, except for the synthetase reaction, essentially irreversible for these kinetic purposes; an arrow from a symbol to the center of another arrow represents the influence of a modifier upon a regulatory enzyme. See text for further discussion. 
formalism that is specifically appropriate for biochemical systems (Savageau, 1976).

$$
\begin{aligned}
& \dot{X}_{1}=\alpha_{1} X_{0}^{g_{10}} X_{n}^{g_{1 n}}-\beta_{1} X_{1}^{h_{11}} \\
& \dot{X}_{2}=\beta_{1} X_{1}^{h_{11}}-\beta_{2} X_{2}^{h_{22}} \\
& \dot{X}_{p}=\alpha_{p} X_{p}^{g_{p p}} X_{p-1}^{g_{p, p}-1}-\beta_{p} X_{p}^{h_{p p}} \\
& \dot{X}_{n}=\alpha_{n} X_{n}^{g_{n \prime}} X_{n-1}^{g_{n, 1}-1}-\beta_{n} X_{n-1}^{h_{n, 1}-1} X_{n}^{h_{n n}} X_{n+1}^{h_{n, n+1}} \\
& \dot{X}_{n+1}=\beta_{n} X_{n-1}^{h_{n, n-1}} X_{n}^{h_{n n}} X_{n+1}^{h_{n, 1}}-\beta_{n+1} X_{n+1}^{h_{n+1, n+1}},
\end{aligned}
$$

where $X_{i}$ is the concentration of the $i$ th metabolite and the dot ( $\left.{ }^{\circ}\right)$ indicates the derivative with respect to time. The net rate of synthesis and the net rate of degradation of a given metabolite are each represented by a product of power laws, one for each reactant or modifier that affects the net rate. The parameters $\alpha_{i}$ and $\beta_{i}$ represent apparent rate constants and $g_{i j}$ and $h_{i j}$ represent apparent kinetic orders. Whenever the net rate of synthesis of one metabolite is identical to the net rate of degradation of the preceding metabolite, the appropriate $\alpha$ and $g$ parameters in equation (1) have been replaced by the corresponding $\beta$ and $h$ parameters.

The corresponding steady-state equations can be obtained in a convenient linear form by setting the time derivatives to zero and taking logarithms:

$$
\begin{aligned}
& \left.\begin{array}{c}
\left(b_{1}-g_{10} y_{0}\right) \\
b_{2} \\
b_{3} \\
\cdot \\
\cdot \\
\left(b_{p}-g_{p p^{\prime}} \cdot y_{p^{\prime}}\right) \\
\cdot \\
\cdot \\
\left(b_{n}-\dot{g}_{n n} \cdot y_{n^{\prime}}\right) \\
b_{n+1}
\end{array}\right]
\end{aligned}
$$

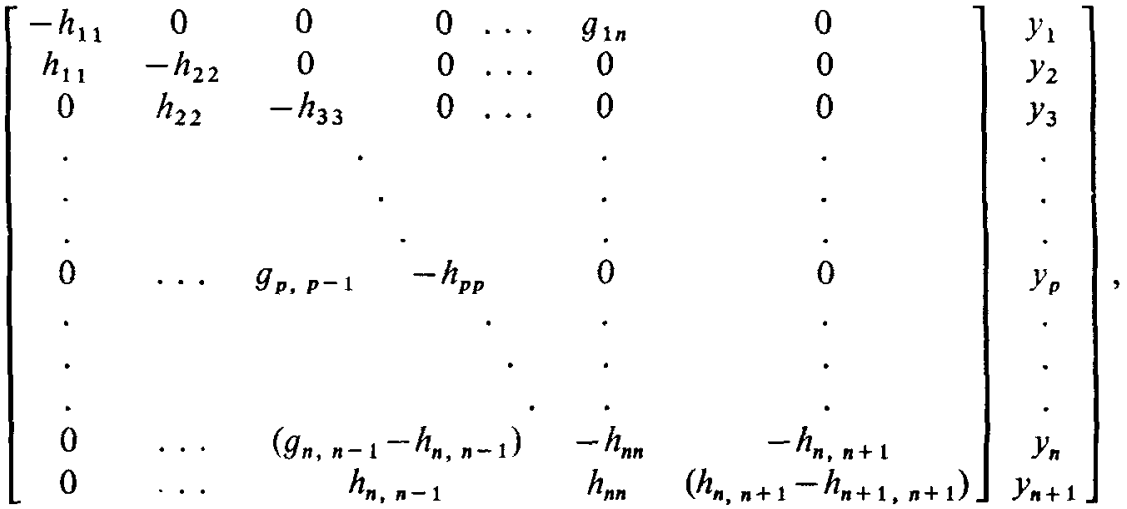


where

$$
\begin{array}{rlrl}
y_{i} & =\log X_{i} & \\
b_{i} & =\log \left(\beta_{i} / \alpha_{i}\right) & & \text { for } i=1, p, n \\
& =\log \left(\beta_{i} / \beta_{i-1}\right) & & \text { for all other } i .
\end{array}
$$

The dependent concentration variables in equation (2) can be solved for in terms of the independent variables $\left(y_{0}, y_{p^{\prime}}, y_{n^{\prime}}\right)$ and the parameters characterizing the system. These solutions form the basis for further analysis as will be described in later sections.

The system represented in Fig. 1 will be compared with an equivalent system lacking feedforward inhibition on the basis of criteria for the functional effectiveness of biosynthetic pathways. These criteria have been discussed elsewhere (Savageau, 1976) and are summarized here briefly: (1). responsiveness to change in the availability of the initial substrate; (2) minimum change in the level of activated end product as it shifts from one steady state to another in response to a change in demand; (3) limited accumulation of metabolic intermediates; (4) reduction of the synthesis of metabolic intermediates when the end product is supplied exogenously; (5) insensitivity to perturbations in the structure of the system itself; (6) stability, and (7) temporal responsiveness to change.

\section{Response to Initial Substrate}

From the steady-state solutions of equation (2) one can calculate the percent change in the concentration of activated end product $\left(X_{n+1}\right)$ for a one-percent change in the concentration of initial substrate $\left(X_{0}\right)$ by taking the partial derivative of $y_{n+1}$ with respect to $y_{0}$. This response of the concentration of activated end product to a change in the steady-state level of the initial substrate is defined as the overall logarithmic gain of the system $\left(L_{n+1}, 0\right)$, which for the system in Fig. 1 is

where

$$
\begin{aligned}
L_{n+1,0} & =\partial y_{n+1} / \partial y_{0} \\
& =g_{10} g_{p, p-1} g_{n, n-1} h_{n n} / \Delta,
\end{aligned}
$$

$$
\begin{aligned}
& \Delta=h_{p-1, p-1} h_{n-1, n-1} h_{n n} h_{n+1, n+1} \\
& \quad+g_{1 n} g_{p, p-1}\left[g_{n, n-1} h_{n, n+1}-h_{n+1, n+1}\left(g_{n, n-1}-h_{n, n-1}\right)\right] .
\end{aligned}
$$

The expression in equation (3) is always positive, indicating that $X_{n+1}$ increases in response to an increase in $X_{0}$, since $g_{1 n}, h_{n, n-1}$, and $h_{n, n+1}$ are the parameters with negative values. Similarly, for the equivalent system without feedforward inhibition $\left(h_{n, n-1}=0\right)$, denoted by the symbols with a zero superscript,

$$
L_{n+1,0}^{0}=g_{10} g_{p, p-1} g_{n, n-1} h_{n n}^{0} / \Delta^{0},
$$


where

$$
\begin{aligned}
\Delta^{0}=h_{p-1, p-1} h_{n-1, n-1} h_{n, 1}^{0} h_{n+1,} & +1 \\
& +g_{1 n} g_{p, p-1}\left[g_{n, n-1}\left(h_{n, n+1}^{0}-h_{n+1, n+1}\right)\right] .
\end{aligned}
$$

For comparisons the systems are required to be identical in every respect except for the properties of the synthetase reaction. Parameters common to the two systems must have identical values wherever possible (internal equivalence). These parameters are represented without superscripts in equation (4). Parameters that characterize the synthetase reaction have different values for the two systems, and these are indicated with a zero superscript in equation (4). The steady-state behavior of the two systems also is required to be identical as far as possible from an external perspective (external equivalence), i.e. the systems will have the same steady-state level of activated end product $\left(X_{n+1}=X_{n+1}^{0}\right)$ in the absence of change, the same response to changes in initial substrate $\left(L_{n+1,0}=L_{n+1,0}^{0}\right)$, and the same response to changes in demand for activated end product $\left({ }^{s} L_{n+1,0} h_{n+1, n+1}\right.$ $={ }^{s} L_{n+1, n+1}^{0}$, see next section). This specification of both internal and external equivalence implies the following constraints among the parameters of the synthetase reactions:

$$
\begin{gathered}
h_{n n}^{0}=h_{n n} g_{n, n-1} /\left(g_{n, n-1}-h_{n, n-1}\right) \\
h_{n, n+1}^{0}=h_{n, n+1} g_{n, n-1} /\left(g_{n, n-1}-h_{n, n-1}\right)
\end{gathered}
$$

$$
\log \beta_{n}^{0}=\left[\log \beta_{n}-\left(h_{n, n-1} / g_{n, n-1}\right) \log \alpha_{n}\right] g_{n, n-1} /\left(g_{n, n-1}-h_{n, n-1}\right)
$$

\section{Response to Demand for Activated End Product}

A change in the demand for activated end product $\left(X_{n+1}\right)$ can be represented by a change in the apparent kinetic order $\left(h_{n+1, n+1}\right)$ for the degradation of $X_{n+1}$, and the response of the system to this change can be represented by the sensitivity of the overall logarithmic gain $\left(L_{n+1,0}\right)$ with respect to $h_{n+1, n+1}$ (Savageau, 1976). By definition,

$$
S_{L_{n+1,0} h_{n+1, n+1}}=\frac{\partial L_{n+1,0}}{\partial h_{n+1, n+1}} \cdot \frac{h_{n+1, n+1}}{L_{n+1,0}}
$$

For the system with feedforward inhibition

$$
\begin{aligned}
& S_{L_{n+1}, 0 h_{n+1, n+1}}=-\left[h_{p-1, p-1} h_{n-1, n-1} h_{n n} h_{n+1, n+1}\right. \\
&\left.-g_{1 n} g_{p, p-1} h_{n+1, n+1}\left(g_{n, n-1}-h_{n, n-1}\right)\right] / \Delta .
\end{aligned}
$$

This expression is always negative, indicating that $X_{n+1}$ decreases in response to an increase in demand for activated end product, since $g_{1 n}, h_{n, n-1}$, and $h_{n, n+1}$ are the only parameters with negative values. The corresponding 
expression for the equivalent system without feedforward inhibition is given by

$$
\begin{aligned}
S_{L^{0} n+1,0} h_{n+1, n+1}=-\left[h_{p-1, p-1} h_{n-1, n-1} h_{n n}^{0} h_{n+1, n+1}\right. \\
\left.-g_{1 n} g_{p, p-1} h_{n+1, n+1} g_{n, n-1}\right] / \Delta^{U}
\end{aligned}
$$

Equivalence of the sensitivities in equations (8) and (9), together with equivalence of the overall logarithmic gains in equations (3) and (4), specifies the constraints in equations (5) and (6). Equivalence of the steady-state levels for the two systems then yields the constraint relation in equation (7).

It should be noted that the synthetase reaction must have some "reversible" component for this type of system to respond to changes in demand for activated end product. This component is most likely the reverse of the normal charging reaction or product inhibition of the synthetase by aminoacyl tRNA, but it also might include a contribution from the "editing" function of synthetases. The latter is a separate function of the synthetases that results in the splitting of incorrect amino acid-tRNA pairs and occasionally the correct amino acid-tRNA pair (Yarus, 1972; Hopfield, 1974; Ninio, 1975).

\section{Accumulation of Metabolic Intermediates}

Although some accumulation of metabolic intermediates will occur when the flux through the pathway is increased in response to either increased availability of the initial substrate or increased demand for activated end product, it should be minimized. Accumulation of metabolic intermediates in an unbranched pathway can make the temporal response of the system sluggish (Koch, 1967) and deplete the limited solvent capacity within the cell.

The responses of the intermediate concentrations $\left(X_{i}\right)$ in the pathway to changes in the steady-state concentration of the initial substrate $\left(X_{0}\right)$ will be represented by the intermediate logarithmic gains $\left(L_{i 0}=\partial y_{i} / \partial y_{0}\right)$, which for the system in Fig. 1 are:

$$
\begin{array}{rlrl}
L_{i 0} & =g_{10} h_{p-1, p-1} h_{n-1, n-1} h_{n n} h_{n+1, n+1} /\left(h_{i i} \Delta\right), & 1 \leq i<p \\
& =g_{10} g_{p, p-1} h_{n-1, n-1} h_{n n} h_{n+1, n+1} /\left(h_{i i} \Delta\right), & p \leq i<n \\
& =-g_{10} g_{p, p-1}\left[g_{n, n-1} h_{n, n+1}-h_{n+1, n+1}\left(g_{n, n-1}-h_{n, n-1}\right)\right] / \Delta, \\
& & i=n .
\end{array}
$$

These expressions, like the overall logarithmic gain, are always positive. Similar expressions can be obtained for the equivalent system lacking feedforward inhibition by setting $h_{n, n-1}$ equal to zero and adding the zero superscript to the parameters $h_{n n}$ and $h_{n, n+1}$. 


$$
\begin{aligned}
L_{i 0}^{0} & =g_{10} h_{p-1, p-1} h_{n-1, n-1} h_{n n}^{0} h_{n+1, n+1} /\left(h_{i i} \Delta^{0}\right), & & 1 \leq i<p \\
& =g_{10} g_{p, p-1} h_{n-1, n-1} h_{n h}^{0} h_{n+1, n+1} /\left(h_{i i} \Delta^{0}\right), & & p \leq i<n \\
& =g_{10} g_{p, p-1} g_{n, n-1}\left(h_{n, n+1}^{0}-h_{n+1, n+1}\right) / \Delta^{0}, & & i=n
\end{aligned}
$$

The parameters with superscripts then can be replaced by the relations in equations (5) and (6) and one finds that

$$
L_{i 0}^{0}=L_{i 0}, \quad 1 \leq i \leq n
$$

The responses of the intermediate concentrations in the pathway to changes in the steady-state demand for activated end product will be represented by the sensitivities of the intermediate logarithmic gains with respect to change in $h_{n+1, n+1}$.

$$
\begin{aligned}
S_{L_{i 0} h_{n+1}, n+1} & =g_{1 n} g_{p, p-1} g_{n, n-1} h_{n, n+1} /\left(h_{n+1, n+1} \Delta\right), \quad 1 \leq i<n \\
& =\frac{g_{n, n-1} h_{p-1, p-1} h_{n-1, n-1} h_{n n} h_{n+1, n+1} h_{n, n+1}}{\left[g_{n, n-1} h_{n, n+1}-h_{n+1, n+1}\left(g_{n, n-1}-h_{n, n-1}\right)\right] \Delta}, \quad i=n
\end{aligned}
$$

These expressions are always positive, which indicates that these intermediate concentrations increase when there is an increase in demand for activated end product. The corresponding expressions for the equivalent system lacking feedforward inhibition can be obtained by setting $h_{n, n-1}$ equal to zero, and by replacing $h_{n n}^{0}$ and $h_{n, n+1}^{0}$ with the constraints in equations (5) and (6). When this is done, one finds

$$
S_{L_{i 0} h_{n+1, n+1}}=S_{L_{10} h_{n+1, n+1}}, \quad 1 \leq i \leq n
$$

Thus, the results in equations (16) and (19) show that the accumulation of metabolic intermediates, either in response to a change in the initial substrate or in response to a change in demand for activated end product, is the same whether or not feedforward inhibition is present.

\section{Response to Addition of Exogenous End Product}

The responses of the dependent concentrations to a change in the steadystate level of exogenous end product $\left(X_{n^{\prime}}\right)$ are given by the following expressions:

$$
\begin{array}{rlrl}
L_{i n^{\prime}} & =\partial y_{i} / \partial y_{n^{\prime}} \\
& =-g_{n n^{\prime}} g_{1 n} h_{p-1, p-1} h_{n-1, n-1}\left(h_{n, n+1}-h_{n+1, n+1}\right) /\left(h_{i i} \Delta\right), & 1 \leq i<p \\
& =-g_{n n^{\prime}} g_{1 n} g_{p, p-1} h_{n-1, n-1}\left(h_{n, n+1}-h_{n+1, n+1}\right) /\left(h_{i i} \Delta\right), & \\
& & & \\
& =-g_{n n}, h_{p-1, p-1} h_{n-1, n-1}\left(h_{n, n+1}-h_{n+1, n+1}\right) / \Delta, & i=n \\
& =g_{n n^{\prime}} \cdot\left(h_{p-1, p-1} h_{n-1, n-1} h_{n n}+g_{1 n} g_{p, p-1} h_{n, n-1}\right) / \Delta, & i=n+1
\end{array}
$$


As expected, the expressions in equations (20) and (21) are always negative, which indicates that all the intermediate concentrations decrease in response to an increase in $X_{n^{\prime}}$, whereas the expressions in equations (22) and (23) are always positive, which indicates that $X_{n}$ and $X_{n+1}$ increase in response to an increase in $X_{n}$.

The equivalent system without feedforward inhibition is described by the same set of equations but with $h_{n, n-1}=0$ and a zero superscript added to the symbols $L_{i n}, h_{n n}$, and $h_{n, n+1}$. These latter equations can be compared with equations (20)-(23) by utilizing the constraint relations in equations (5) and (6) and by taking the ratio of the corresponding equations. Thus,

$$
\begin{aligned}
& \frac{L_{i n^{\prime}}}{L_{i n^{\prime}}^{0}}=\frac{g_{n, n-1}\left(h_{n, n+1}-h_{n+1, n+1}\right)}{g_{n, n-1}\left(h_{n, n+1}-h_{n+1, n+1}\right)+h_{n+1, n+1} h_{n, n-1}}<1 . \quad 1 \leq i \leq n \\
& =\frac{h_{p-1, p-1} h_{n-1, n-1} h_{n n}+g_{1 n} g_{p, p-1} h_{n, n-1}}{h_{p-1, p-1} h_{n-1, n-1} h_{n n}}>1, \quad i=n+1 .
\end{aligned}
$$

The magnitudes of the changes in concentration for the intermediates are always less in the system with feedforward inhibition, implying that the synthesis of these metabolic intermediates is less effectively spared in response to addition of exogenous end product. The magnitude of the increase in $X_{n}$ is less, while that for $X_{n+1}$ is greater, in the system with feedforward inhibition. The extra increase in $X_{n+1}$ with feedforward inhibition is due to the reduced sparing of endogenous synthesis of $X_{n}$ rather than to greater utilization of $X_{n}$. [This is in contrast to the suggestion, based upon preliminary analysis, that feedforward inhibition causes a more efficient funneling of the exogenous end product into the aminoacyl-tRNA pool (Savageau, 1972).]

\section{Response to Perturbations in the Structure of the System}

The structure (or relatively fixed part) of the system is specified by the parameter values in equation (1). The response of the system to perturbations in the structure of the system itself can be determined by calculating the sensitivity of $X_{n+1}$ or of the overall logarithmic gain, with respect to the parameter of interest. For example,

$$
\begin{aligned}
S_{L_{n+1,0} h_{n-1, n-1}} & =\frac{\partial L_{n+1,0}}{\partial h_{n-1, n-1}} \frac{h_{n-1, n-1}}{L_{n+1,0}} \\
& =-h_{p-1, p-1} h_{n-1, n-1} h_{n n} h_{n+1, n+1} / \Delta
\end{aligned}
$$

and for the equivalent system without feedforward inhibition,

$$
\begin{aligned}
S_{L^{0} n+1,0} h_{n-1, n-1} & =-h_{p-1, p-1} h_{n-1, n-1} h_{n n}^{0} h_{n+1, n+1} / \Delta^{0} \\
& =-h_{p-1, p-1} h_{n-1, n-1} h_{n n} h_{n+1, n+1} / \Delta .
\end{aligned}
$$


Thus, the two systems are equally sensitive to a change in the structural determinant $h_{n-1, n-1}$. Similar calculations show that the two systems are equally sensitive to changes in the other parameters they have in common.

\section{Stability}

The descriptive equations (1) can be linearized about a steady-state operating point (Savageau, 1976). The local stability of this state then can be determined by examining the roots of the characteristic equation,

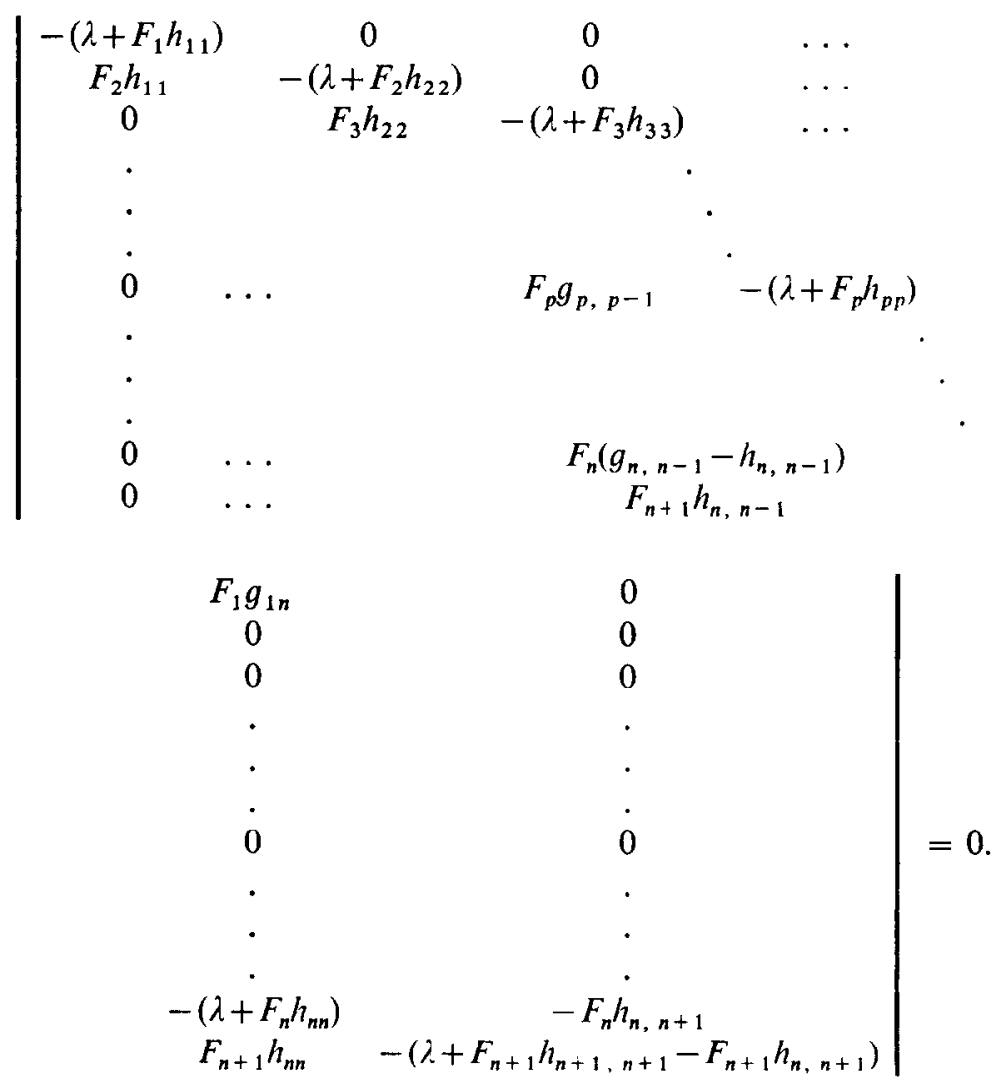

Significant differences in value among the corresponding kinetic parameters for the uncontrolled reactions in a scquence such as that in Fig. 1 are known to effectively shorten the path length from a kinetic perspective, and the system can be represented by fewer equations in which the corresponding 
kinetic parameters are more nearly identical (Savageau, 1975). Therefore, without loss of generality one can consisder

$$
\begin{aligned}
& F_{i}=F \quad \text { for all } i, \\
& h_{i i}=h \quad \text { for all } i<n, \text { and } \\
& h_{n n}^{0}=h_{n+1, n+1}-h_{n, n+1}^{0}=h
\end{aligned}
$$

In spite of these simplifications, there is no evident solution of equation (28) whether or not feedforward inhibition is considered. However, these solutions can be obtained by a perturbation analysis based upon the solution for a system without feedforward inhibition $\left(h_{n, n-1}=0\right)$ and without the editing function (or the reverse reaction) of the synthetase $\left(h_{n, n+1}=0\right)$. Under these latter conditions, equation (28) reduces to

$$
(\lambda+F h)^{n}-g(F h)^{n}=0
$$

where

$$
g=g_{1 n} g_{p, p-1} g_{n, n-1} h_{l} \cdots
$$

and the solution is given by

$$
\lambda_{0}=F h\left[(-1)^{1 / n}(-g)^{1 / n}-1\right] .
$$

The characteristic equation for the system with the editing function (or the reverse reaction) of the synthetase $\left(h_{n, n+1}^{0} \neq 0\right)$, but still lacking feedforward inhibition $\left(h_{n, n-1}=0\right)$, is given by

$$
(\lambda+F h)^{n}-g(F h)^{n}+F^{2} h_{n, n+1}^{0} h(\lambda+F h)^{n-2}=0 .
$$

Consider a solution of the form

$$
i=\lambda_{0}+\lambda_{1} \varepsilon+\lambda_{2} \varepsilon^{2}+
$$

where

$$
\varepsilon=-F h_{n, n+1}^{0} i n
$$

Substitution of this solution into equation (32) and setting the coefficient for each power of $c$ to zero yields the following series of equations

$$
\begin{aligned}
& \left(\lambda_{0}+F h\right)^{n}-g(F h)^{n}=0 \\
& \left(\lambda_{0}+F h\right) \lambda_{1}-F h=0 .
\end{aligned}
$$

Equation (34) yields the zero-order approximation $i_{0}$. The first-order approximation is obtained from equation (35):

$$
\lambda_{1}=F h /\left(\lambda_{0}+F h\right) \text {. }
$$


If $\varepsilon$ is small, the higher-order approximations can be neglected and the solution is given by

$$
\lambda=\lambda_{0}+\lambda_{1} \varepsilon
$$

The real parts of these solutions must be negative for stability. If the reference system is chosen to be on the boundary of stability, i.e., $\operatorname{Re}\left(\lambda_{0}\right)=0$ for the root with the largest real part, then

$$
g=-\sec ^{n}(\pi / n)
$$

and for the solution obtained by perturbation analysis

$$
\begin{aligned}
\operatorname{Re}(j) & =\varepsilon \operatorname{Re}\left(\lambda_{1}\right) \\
& =\varepsilon \cos ^{2}(\pi / n)>0
\end{aligned}
$$

for the root with the largest real part. Thus, addition of an editing function (or the reverse component) to the synthetase reaction decreases the stability of this system in comparison to the reference system.

The same perturbation analysis can be performed for the equivalent system with feedforward inhibition $\left(h_{n, n-1} \neq 0\right)$ and an editing function (or a reverse component) for the synthetase reaction $\left(h_{n, n+1} \neq 0\right)$. Its characteristic equation can be simplified by using equations (5) and (6) and the result is written

$$
\begin{aligned}
& {\left[(\lambda+F h)^{n}-g(F h)^{n}\right]+F^{2} h_{n, n+1}^{0} h(\lambda+F h)^{n-2}} \\
& \quad-\frac{h_{n, n-1}}{g_{n, n-1}} F h\left[(\lambda+F h)^{n-1}-g(F h)^{n-1}\right] \\
& \quad+\frac{h_{n, n-1}}{g_{n, n-1}} F h_{n, n+1}^{0}(\lambda+F h)^{n-2}[(\lambda+F h)-2 F h]=0
\end{aligned}
$$

Consider a power-series solution in two variables:

$$
\lambda=\lambda_{0}+\lambda_{11} \varepsilon+\lambda_{12} \delta+\lambda_{21} \varepsilon^{2}+\lambda_{22} \delta^{2}+\lambda_{23} \varepsilon \delta+\ldots,
$$

where

and

$$
\varepsilon=-F h_{n, n+1}^{0} / n
$$

$$
\delta=-h_{n, n-1} F h /\left(n g_{n, n-1}\right) .
$$

Substitution of this solution into equation (40) and setting the coefficient for each power of $\varepsilon$ and $\delta$ to zero yields the following series of equations

$$
\begin{aligned}
& \left(\lambda_{0}+F h\right)^{n}-g(F h)^{n}=0 \\
& \left(\lambda_{0}+F h\right) \lambda_{11}-F h=0 \\
& \left(\lambda_{0}+F h\right)^{n-1} \lambda_{12}+\left[\left(\lambda_{0}+F h\right)^{n-1}-g(F h)^{n-1}\right]=0 .
\end{aligned}
$$


These equations give the zero- and first-order terms in the approximation, and if $\varepsilon$ and $\delta$ are small, the higher-order terms can be neglected. The approximate solution then is given by

$$
\lambda=\lambda_{0}+\lambda_{11} \varepsilon+\lambda_{12} b \text {. }
$$

Again, if the reference system is chosen to be on the boundary of stability, i.e. $\operatorname{Re}\left(\lambda_{0}\right)=0$ for the root with the largest real part, then $q=-\sec ^{n}(\pi / n)$ and for the solution obtained by perturbation analysis

$$
\begin{aligned}
\operatorname{Re}(\lambda) & =\varepsilon \operatorname{Re}\left(\lambda_{11}\right)+\delta \operatorname{Re}\left(\lambda_{12}\right) \\
& =\varepsilon \cos ^{2}(\pi / n)+\delta \operatorname{Re}\left[(-1)^{1 / n}(-g)^{1 / n}-1\right] \\
& =\varepsilon \cos ^{2}(\pi / n)
\end{aligned}
$$

for the root with the largest real part.

Thus, to a first approximation the systems with and without feedforward inhibition have the same degree of stability when $g=-\sec ^{n}(\pi / n)$. Feedforward inhibition is a destabilizing influence when $g<-\sec ^{n}(\pi / n)$. It is a stabilizing influence when $g>-\sec ^{n}(\pi / n)$, but the degree of stabilization decreases with $n$, the length of the pathway. These conclusions based on analysis also are supported by computer simulation of the non-linear systems.

The techniques are those discussed elsewhere (Savageau, 1976). Systems represented by Fig. I and an equivalent system lacking feedforward inhibition have been examined. The systems are in a steady state with concentrations normalized to unity prior to $t=0$. At time zero the initial substrate concentration for each system is suddenly increased and maintained at a constant elevated level for the subsequent time period. The response of the concentration of the activated end product $\left(X_{n+1}\right)$ is shown as a function of time.

The destabilizing influence of feedforward inhibition is shown in Fig. 2 for a system with $n=4$ and $g=-6$. The system without feedforward inhibition is unstable. As the strength of feedforward inhibition is increased in a series of equivalent systems, the unstable oscillations grow at a faster rate for each successive system. If, however, the strength of feedforward inhibition is increased sufficiently $\left(h_{43} \leq-0.5\right)$, a stabilizing tendency appears. This also is found analytically if the higher-order terms are included in the expansion of the roots of the characteristic equation. If $h_{43} \leq-16 \cdot 0$, this stabilizing tendency is sufficient to make the system with feedforward inhibition stable.

The stabilizing influence of feedforward inhibition is shown in Fig. 3 for a system with $n=4$ and $g=-3 \cdot 74$. The system without feedforward inhibition is slightly unstable. As the strength of feedforward inhibition is increased in a series of equivalent systems, each succeeding system become more stable. It also is clear that the increment in stability for each doubling 


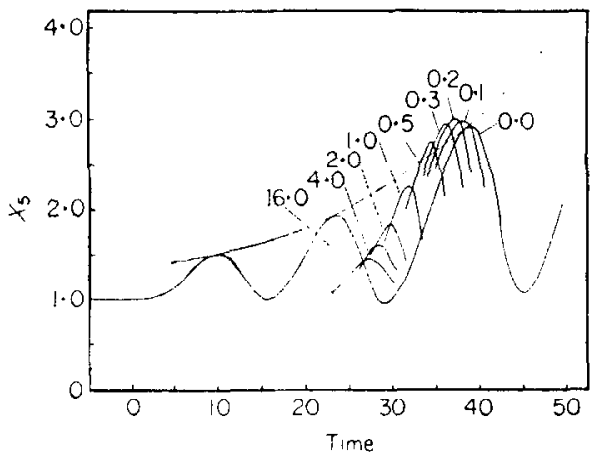

FiG. 2. Destabilizing effect of feedforward inhibition. The model in Fig. 1 is simulated with $n=4, g=-6$ [i.e. $g<\sec ^{n}(\pi / n)$ ], and various strengths of feedforward inhibition $\left(-h_{43}\right)$ as indicated. The independent concentration variable $X_{0}$ is perturbed at $t=0$. For clarity only the progression of the third peak is shown. See text for further discussion.

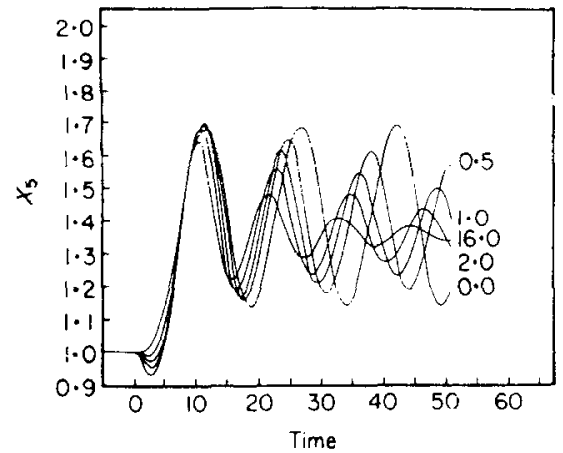

FIG. 3. Stabilizing effect of feedforward inhibition. The conditions are the same as in Fig. 2 except $g=-3 \cdot 74$.

of the strength of feedforward inhibition becomes progressively smaller. Essentially, no further increase in stability can be achieved by increasing $h_{43}$ beyond -- 16.0. Similar phenomena are shown in Figs. 4 and 5 for a longer pathway with $n=9$. Comparing the results shown in Figs 3 and 5 reveals that the same increment in the strength of feedforward inhibition produces a greater stabilizing influence for the shorter pathway.

\section{Temporal Responsiveness}

The system represented in Fig. 1 (with $n=4$ ) and an equivalent one lacking feedforward inhibition have been examined under conditions ensuring stability by using the computer simulation techniques referred to in section 8 . 


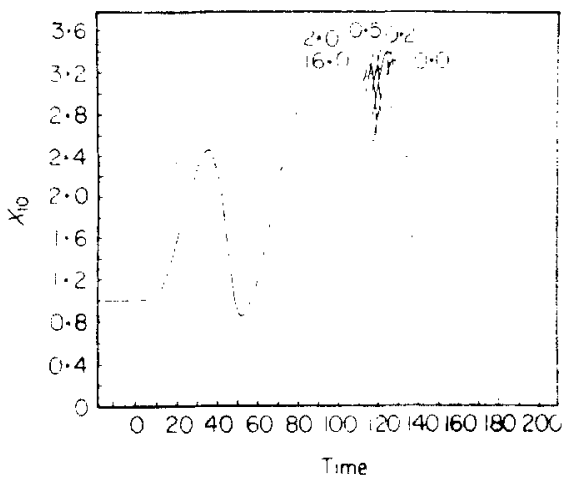

FIG. 4. Destabilizing effect of feedforward inhibition. The model in Fig. 1 is simulated with $n=9, g=-1.8$, and various strengths of feedforward inhibition $\left(\cdot h_{98}\right)$ as indicated. The independent concentration variable $X_{0}$ is perturbed at $t=0$. For clarity only the progression of the third peak is shown. See text for further discussion.

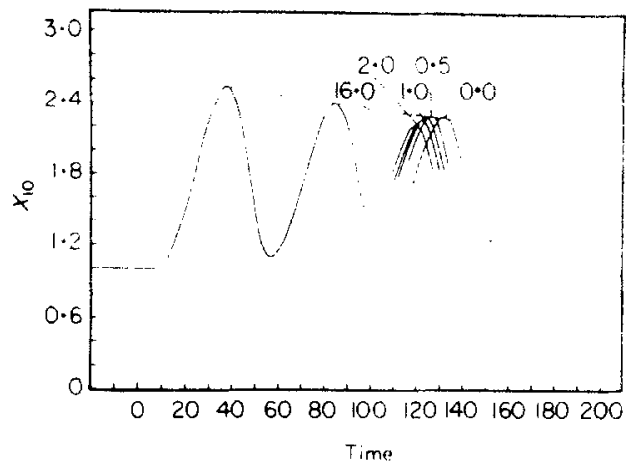

Fig. 5. Stabilizing effect of feedforward inhibition. The conditions are the same as in Fig. 4 except that $g=-1 \cdot 4$.

A typical result is shown in Fig. 6. The system with only feedback inhibition exhibits a smooth increase in $X_{5}$ and then a slight overshoot before achieving the new steady state. The system that possesses feedforward inhibition as well has a different temporal response. The concentration of its activated end product exhibits a "false start" before increasing toward the new steady state. It also experiences a slightly more exaggerated overshoot before finally reaching the new steady state. The response time, as measured by the time for half-maximal response or the time to reach 90 per cent of the maximal response and remain within 10 per cent of it, is generally lengthened by feedforward inhibition. In this particular comparison the response time is between 20 and 50 per cent greater than that of the system without this mechanism, depending upon the method of measurement. 


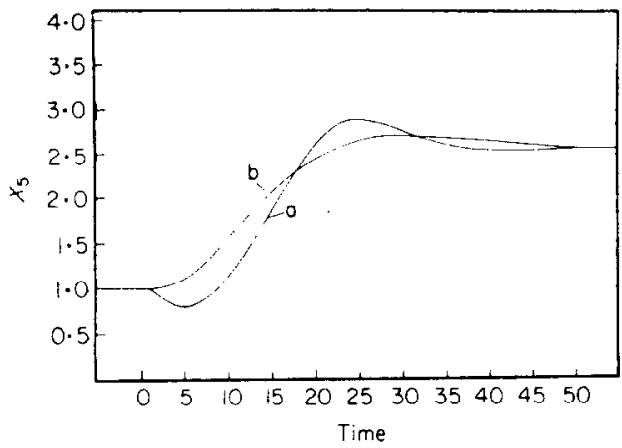

FIG. 6. Temporal responsiveness of equivalent systems with and without feedforward inhibition. (a) The model in Fig. 1 is simulated with $n=4, g=-0.4$ and $h_{43}=-4 \cdot 0$, and (b) an equivalent model is simulated without feedforward inhibition. The independent concentration variable $X_{0}$ is perturbed at $t=0$. See text for further discussion.

\section{Response to Addition of Exogenous Intermediates}

The responses of the dependent concentrations to a change in concentration of an exogenous intermediate $\left(X_{p}\right)$ are given by the following cxpressions:

$$
\begin{aligned}
& L_{i p^{\prime}}=-g_{p p^{\prime}} g_{1 n} h_{p-1, p-1}\left[g_{n, n-1} h_{n, n+1}-h_{n+1, n+1}\left(g_{n, n-1}\right.\right. \\
& \left.\left.-h_{n, n-1}\right)\right] /\left(h_{i i} \Delta\right), \quad 1 \leq i<p \\
& =g_{p p}, h_{p-1, p-1} h_{n-1, n-1} h_{n n} h_{n+1, n+1} /\left(h_{i i} \Delta\right), \quad p \leq i<n \\
& =-g_{p p}, h_{p-1, p-1}\left[g_{n, n-1} h_{n, n+1}-h_{n+1, n+1}\left(g_{n, n-1}\right.\right. \\
& \left.\left.-h_{n, n-1}\right)\right] / \Delta, \quad i=n \\
& =g_{p p^{\prime}} g_{n, n-1} h_{p-1, p-1} h_{n n} / \Delta, \quad i=n+1 .
\end{aligned}
$$

All the concentrations preceding $X_{p}$ decrease, whereas all those from $X_{p}$ on increase. in response to an increase in $X_{p^{\prime}}$.

The same set of equations, but with $h_{n, n-1}=0$, and $h_{n n}, h_{n, n+1}$ and $\Delta$ replaced by $h_{n n}^{0}, h_{n, n+1}^{0}$ and $\Delta^{0}$, respectively, describes the equivalent system without feedforward inhibition. By utilizing the constraints in equations (5) and (6), one finds that

$$
L_{i p^{\prime}}^{0}=L_{i p^{\prime}}, \quad 1 \leq i \leq n+1 .
$$

Thus, feedforward inhibition has no effect on the system's response to additions of exogenous intermediates. In particular, the concentration of activated end product must increase in response to the addition of an exogenous intermediate, contrary to the behaviour that has been attributed to this type of model of feedforward inhibition in the arginine pathway of Chl. reinhardi (Sussenbach \& Strijkert, 1969). [See also an earlier criticism in Savageau (1972).] 
However, if the model of Sussenbach \& Strijkert (1969) is modified by the inclusion of an alternative reaction for the utilization of the end product $X_{n}$, a model equivalent to that shown in Fig. 7 is obtained that is capable of explaining their experimental results. In fact, the model in Fig. 7 corresponds more closely than the original model of Sussenbach \& Strijkert (1969) (and also the model in Fig. 1) to the actual situation in many biosynthetic pathways for amino acids. The analysis is very similar to that given above except that the parameters $x_{n+1}, g_{n+1, n}, g_{n+1, n-1}$ and $g_{n+1, n+1}$ can no longer be

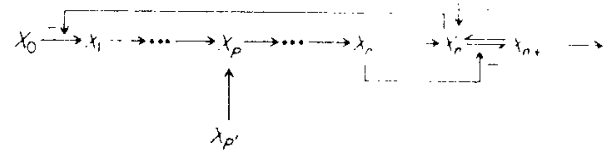

Fig. 7. Model of a pathway for the biosynthesis of an amino acid that has an alternative fate to protein synthesis. The model is otherwise identical to that in Fig. 1. See text for further discussion.

replaced by $\beta_{n}, h_{n n}, h_{n, n}$, and $h_{n, n+1}$, respectively. The change in the concentration of activated end product $X_{n+1}$ resulting from an addition of exogenous intermediate $X_{p}$, is given by

$$
L_{n+1, p}=-g_{p p}, h_{p-1, p-1}\left[g_{n+1, n}\left(g_{n, n-1}-h_{n, n-1}\right)+h_{n n} g_{n+1, n-1}\right] / \Delta^{\prime}
$$

where

$$
\begin{array}{r}
\Delta^{\prime}=\left\{h_{p-1, p-1} h_{n-1, n-1}\left[g_{n+1, n} h_{n, n+1}-h_{n n}\left(g_{n+1, n+1}-h_{n+1, n+1}\right)\right]\right. \\
+g_{1 n} g_{p, p-1}\left[\left(g_{n, n-1}-h_{n, n-1}\right)\left(g_{n+1, n+1}-h_{n+1, n+1}\right)\right. \\
\left.\left.+h_{n, n+1} g_{n+1, n-1}\right]\right\}
\end{array}
$$

For a stable system the denominator will be positive. The numerator, on the other hand, can be either positive or negative depending on the particular values of the parameters involved. If, for instance,

then

$$
g_{n, n-1}>h_{n, n-1}-\left(h_{n n} / g_{n+1, n}\right) g_{n+1, n-1},
$$

$$
L_{n+1, p^{\prime}}=\partial y_{n+1} / \partial y_{p^{\prime}}>0 .
$$

That is, if the apparent kinetic order for the rate of synthesis of end product with respect to the penultimate product is sufficiently large, then the concentration of activated end product $X_{n+1}$ always will increase in response to an increase in exogenous intermediate $X_{p^{\prime}}$. However, if

then

$$
g_{n, n-1}<h_{n, n-1}-\left(h_{n n} / g_{n+1, n}\right) g_{n+1, n+1}
$$

$$
L_{n+1, p^{\prime}}=\partial y_{n+1} / \partial y_{p^{\prime}}<0
$$


This implies that a sufficiently small apparent kinetic order will ensure a decrease in $X_{n+1}$ for an increase in $X_{p^{\prime}}$. When this latter constraint is satisfied, the model in Fig. 7 is capable of explaining the observed reduction in growth rate of Chl. reinhardi upon addition of citrulline.

The model in Fig. 7 appears to be consistent with a set of similar observations in Neurospora (Nazario, 1967). The addition of exogenous citrulline does not lead to a reduction in growth rate in the wild-type organism. Presumably the constraint in equation (53) is satisfied for this organism. However, when an arginine auxotroph lacking the enzyme argininosuccinase is grown on limiting levels of arginine, the addition of citrulline does reduce the growth rate. For this mutant the apparent kinetic order of argininosuccinase with respect to its substrate is zero, i.e. there is no increase in the rate for an increase in substrate concentration. The constraint in equation (54) then applies for the mutant, and as predicted by the model in Fig. 7, addition of exogenous citrulline causes a reduction in the level of arginyl tRNA and a slower growth rate.

Similarly, in $E$. coli the addition of exogenous citrulline normally does not lead to a reduction in growth rate. However, in an arginine bradytroph having reduced argininosuccinase activity, the addition of exogenous citrulline causes a decrease in growth rate ( $R$. Bockrath, unpubl. obs.). In this instance, argininosuccinase activity is not completely lacking; there is merely a quantitative change in the kinetic parameters of the enzyme. Nevertheless, a quantitative change in parameter values can produce a qualitative change in the behavior of the intact system, as indicated by the conditions in equations (53) and (54). Although $E$. coli normally lacks the enzyme arginase, which provides the alternative to aminoacylation in $\mathrm{Chl}$. reinhardi and Neurospora, it does possess a pathway, leading to the synthesis of putrescine, that provides a fate for arginine other than protein synthesis (Morris \& Pardee, 1966).

\section{Discussion}

While there is some experimental evidence for and some against feedforward inhibition by the penultimate product, the results of the analysis in sections 3-9 indicate that this mechanism is unlikely to play a significant role in regulating biosynthetic pathways at the level of enzyme activity. On the basis of criteria 1,2,3 and 5 in section 2, the functional effectiveness of the system with feedforward inhibition was found to be the same as that of the equivalent system without this extra mechanism. On the basis of criteria 4 and 7 , addition of feedforward inhibition actually tends to decrease the effectiveness of the system. With regard to criterion 6 , stability, feedforward 
inhibition can be a stabilizing influence, but the influence is less for long pathways in which stability problems are most pronounced (Savageau, 1975).

These are the same criteria that previously were used to show that endproduct inhibition is an optimal design for the control of biosynthetic pathways by feedback inhibition of enzymatic activity (Savageau, 1974, 1975) and thus to provide an explanation for the nearly universal occurrence of this mechanism in nature.

Could these conclusions be wrong because an inappropriate model was chosen to represent the reaction mechanism of the aminoacyl-tRNA synthetase? The overall behavior of most enzyme-catalyzed reactions is accurately described by quasi-steady-state kinetics in which there is a single rate-limiting process and all other elementary reactions that comprise the mechanism are assumed to occur more rapidly and be in quasi-steady state (Segel, 1975). Many aminoacyl-tRNA synthetases appear to fall within this group (Eigner \& Loftfield, 1974; Midelfort \& Mehler, 1974; Santi et al., 1974), and the analysis presented in the preceding sections is valid for these.

For some synthetases there might be several kinetically comparable dominant steps in the reaction mechanism, although I know of no experimental evidence for this. An cxamination of models representing this possibility shows that the position of the step subject to feedforward inhibition in such a mechanism does affect system behavior. However, in no case has the behavior been found to be more effective than that of the equivalent system without feedforward inhibition. This conclusion is based on analysis and computer simulation, but it also can be appreciated from the following intuitive argument concerning stability.

Stability is related to the number of kinetically comparable dominant steps in a closed cause-effect loop: the smaller the number, the greater the degree of stability (other things being equal). For example, in the system without feedforward inhibition there are $n$ such steps leading from $X_{1}$ and $X_{n}$ and then back to $X_{1}$ via feedback inhibition. For the equivalent system in which the conversion of $X_{n}$ to $X_{n+1}$ is subject to feedforward inhibition by $X_{n-1}$. there is no essential change in the number of kinetically comparable dominant steps in the cause-effect loops. The number from $X_{n}$ to $X_{1}$ to $X_{n-1}$ is unchanged, and the number from $X_{n-1}$ to $X_{n}$ either via synthesis of $X_{n}$ or via inhibition of $X_{n}$ degradation is one. However, if a more distal step (say $X_{n+j-1} \rightleftharpoons X_{n+j}$, where $j>1$ ) in the synthetase reaction mechanism is subject to feedforward inhibition by $X_{n-1}$, then there would be an increase in the number of kinetically comparable dominant steps. The number of such steps from $X_{n}$ to $X_{1}$ to $X_{n-1}$ is still unchanged, as is the number from $X_{n-1}$ to $X_{n}$ via synthesis of $X_{n}$, but the number from $X_{n-1}$ to $X_{n}$ via inhibition of $X_{n+j-1}$ degradation has increased to $j$ and consequently the degree of 
stability has decreased. Thus, the conclusions of this article would not be altered significantly if such more complex mechanisms for the synthetase reaction were to be considered.

Feedforward inhibition by the penultimate product may have no physiological function: competition for a synthetase between the cognate amino acid and its (structurally similar) immediate precursor may be unavoidable for some structural reason and have nothing to do with regulation of the biosynthetic pathway. The suggestion that such competition arises from structural considerations and that it is not selected for regulation of the biosynthetic pathway is further consistent with the following observation. Histidinol, the immediate precursor of histidine, competitively inhibits the histidyl-tRNA synthetase not only in S. typhimurium (Ames \& Hartman, 1961) where there is a functional histidine biosynthetic pathway, but also in human cells where there is not (Hansen, Vaughan \& Wang, 1972).

Feedforward inhibition may have some physiological function, but not that of regulating an unbranched biosynthetic pathway at the level of enzyme activity. One possible function involves the differential signal between $X_{n+1}$ and $X_{n}$ that is generated by feedforward inhibition. The analysis in section 6 showed that an addition of exogenous end product causes an increase in $X_{n+1}$ that is greater, in relation to the increase in $X_{n}$, than would be the case for the equivalent system without feedforward inhibition. On the other hand, the relative changes in $X_{n+1}$ and $X_{n}$ in response to changes in substrate or in demand for activated end product are the same whether or not the system possesses feedforward inhibition. Thus, feedforward inhibition can signal that an addition of exogenous end product has occurred-an extra- rather than intra-cellular change. Presumably this differential in response between $X_{n-1}$ and $X_{n}$ could be used to the cell's advantage by some other control system that recognizes both of these metabolic signals. Examples might include the systems that control biosynthetic pathways by modulating enzyme synthesis; in certain of these cases control is known to involve both aminoacyl tRNA and end product as metabolic signals (see review by Savageau, 1979).

Another possible function has to do with the distribution of metabolic flux among branched biosynthetic pathways. In the preceding section a model was presented that includes both feedback and feedforward inhibition, as well as alternative fates for the end product $X_{n}$, and that is capable of explaining the diverse physiological behavior reported for the arginine biosynthetic pathway in several different organisms. It can be predicted from this model that, when the arginine level is increased via endogenous synthesis, a greater percentage of it will be diverted to fates other than protein synthesis. On the other hand, when the intracellular arginine level is increased by 
exogenous addition of arginine, a greater percentage of it will be diverted to protein synthesis. Experiments using tracer levels of radioactive arginine supplied exogenously, or endogenously via externally added citrulline, have established the percentage of arginine normally diverted to each of its fates in Neurospora cells grown in minimal medium (Subramanian, Weiss \& Davis, 1973). Arginine labeled by either method appears to be used preferentially for protein synthesis. To test the above prediction derived from the model, large (non-tracer) amounts of exogenous arginine or citrulline would have to be added and the percentage of arginine diverted to its various fates compared with the basal pattern of flux established by Subramanian et al. (1973).

This work was supported in part by a grant from the National Science Foundation (BMS 75-01591 and PCM78-07635) and a fellowship from the John Simon Guggenheim Memorial Foundation. Computer facilities were provided by the University of Michigan Medical School. I thank A. M. Kotre for helpful criticism of the manuscript.

\section{REFERENCES}

Ames, B. N. \& HaRtman, P. E. (1961). In Molecular Basis of Neoplasia, Fifteenth Symposium of Fundamental Cancer Research, pp. 322-345. Austin: University of Texas Press.

BArthelmess, I. B., Curtis, C. F. \& Kacser, H. (1974). J. mol. Biol. 87, 303.

Charlier, J. \& Gerlo, E. (1976). Eur. J. Biochem. 70, 137.

Eigner, E. A. \& LofTfield, R. B. (1974). In Methods in Enzymology (S. P. Colowick \& N. O. Kaplan, eds) Vol XXIX, pp. 601-619. New York: Academic Press.

Hansen, B. S., Vaughan, M. H. \& Wang, L.-J. (1972). J. biol. Chem. 247, 3854.

Hopfield, J. J. (1974). Proc. Natn. Acad. Sci. U.S.A. 71, 4135.

KocH, A. L. (1967). J. theor. Biol. 15, 75.

Midelfort, C. F. \& Mehler, A. H. (1974). In Methods in Enzymology (S. P. Colowick \& N. O. Kaplan, eds) Vol. XXIX, pp. 627-642. New York: Academic Press.

MorRIS, D. R. \& PARdeE, A. B. (1966). J. biol. Chem. 241, 3129.

NAZARIO, M. (1967). Biochim. biophys. Acta 145, 138.

NinIO, J. (1975). Biochimie 57, 517.

SANTI, D. V., WeBster, R. W., JR. \& Cleland, W. W. (1974). In Methods in Enzymology (S. P. Colowick \& N. O. Kaplan, eds) Vol. XXIX, pp. 620-627. New York: Academic Press.

Savageau, M. A. (1972). Curr. Top. Cell. Reg. 6, 63.

Savageau, M. A. (1974). J. mol. Evol. 4, 139.

Savageau, M. A. (1975). J. mol. Evol. 5, 199.

Savageau, M. A. (1976). Biochemical Systems Analysis: a Study of Function and Design in Molecular Biology. Reading, Mass.: Addison-Wesley.

Savageau, M. A. (1978). In Biological Regulation and Development (R. F. Goldberger, ed. ), pp. 57-108. New York: Plenum Press.

SEGEL, I. H. (1975). Enzyme Kinetics: Behavior and Analysis of Rapid Equilibrium and Steady-State Enzyme Systems. New York: Wiley.

Spurgeon, S. L. \& MatchetT, W. H. (1977). J. Bacteriol. 129, 1303.

Subramanian, K. N., Weiss, R. L. \& Davis, R. H. (1973). J. Bacteriol. 115, 284.

Sussenbach, J. S. \& StriJKert, P. J. (1969). Eur. J. Biochem. 8, 403.

Williams, A. L., Yem, D. W., MCGinnis, E. \& Williams, L. S. (1973). J. Bacteriol, 115, 228.

Yarus, M. (1972). Proc. Natn. Acad. Sci. U.S.A. 69, 1915. 